\title{
Surprising variations in the rotation of the chemically peculiar stars CU Virginis and V901 Orionis ^
}

\author{
Z. Mikulášek ${ }^{1,2}$, J. Krtička ${ }^{1}$, G. W. Henry ${ }^{3}$, J. Janík ${ }^{1}$, J. Zverko $^{4}$, J. Žižňovský ${ }^{5}$, M. Zejda ${ }^{1}$, J. Liška ${ }^{1}$, P. Zvěřina $^{1}$, \\ D. O. Kudrjavtsev ${ }^{6}$, I. I. Romanyuk ${ }^{6}$, N. A. Sokolov ${ }^{7}$, T. Lüftinger ${ }^{8}$, C. Trigilio ${ }^{9}$, C. Neiner ${ }^{10}$, and S. N. de Villiers ${ }^{11}$ \\ 1 Department of Theoretical Physics and Astrophysics, Masaryk University, Kotlářská 2, CZ 611 37, Brno, Czech Republic \\ e-mail: mikulas@physics.muni.cz \\ 2 Observatory and Planetarium of Johann Palisa, VŠB - Technical University, Ostrava, Czech Republic \\ 3 Center of Excellence in Information Systems, Tennessee State University, Nashville, Tennessee, USA \\ 4 Tatranská Lomnica 133, SK 059 60, Slovak Republic \\ 5 Astronomical Institute of Slovak Academy of Science, Tatranská Lomnica, Slovak Republic \\ 6 Special Astrophysical Observatory of RAS, Nizhnij Arkhyz, Russia \\ 7 Central Astronomical Observatory at Pulkovo, Russia \\ 8 Institute for Astronomy of the University of Vienna, Vienna, Austria \\ 9 INAF - Osservatorio Astrofisico di Catania, Italy \\ ${ }_{11}$ LESIA, Observatoire de Paris, CNRS, UPMC, Université Paris Diderot, 5 place Jules Janssen, 92190 Meudon Cedex, France \\ 11 Private Observatory, 61 Dick Burton Road, Plumstead, Cape Town, South Africa
}

Received 29 July 2011/ Accepted 20 September 2011

\section{ABSTRACT}

\begin{abstract}
Context. The majority of magnetic chemically peculiar $(\mathrm{mCP})$ stars exhibit periodic light, radio, spectroscopic and spectropolarimetric variations that can be adequately explained by the model of a rigidly rotating main-sequence star with persistent surface structures. CU Vir and V901 Ori belong among these few $\mathrm{mCP}$ stars whose rotation periods vary on timescales of decades.

Aims. We aim to study the stability of the periods in CU Vir and V901 Ori using all accessible observational data containing phase information.

Methods. We collected all available relevant archived observations supplemented with our new measurements of these stars and analysed the period variations of the stars using a novel method that allows for the combination of data of diverse sorts.

Results. We found that the shapes of their phase curves were constant during the last several decades, while the periods were changing. At the same time, both stars exhibit alternating intervals of rotational braking and acceleration. The rotation period of CU Vir was gradually shortening until the year 1968, when it reached its local minimum of $0.52067198 \mathrm{~d}$. The period then started increasing, reaching its local maximum of $0.5207163 \mathrm{~d}$ in the year 2005. Since that time the rotation has begun to accelerate again. We also found much smaller period changes in CU Vir on a timescale of several years. The rotation period of V901 Ori was increasing for the past quarter-century, reaching a maximum of $1.538771 \mathrm{~d}$ in the year 2003, when the rotation period began to decrease.

Conclusions. We propose that dynamical interactions between a thin, outer magnetically-confined envelope, braked by the stellar wind, with an inner, faster rotating stellar body is able to explain the observed rotational variability. A theoretically unexpected alternating variability of rotation periods in these stars would remove the spin-down time paradox and brings a new insight into structure and evolution of magnetic upper-main-sequence stars.
\end{abstract}

Key words. stars: chemically peculiar - stars: variables: general - stars: individual: CU Vir - stars: individual: V901 Ori stars: rotation

\section{Introduction}

In addition to mass, all stars inherit a fraction of the angular momentum of the mother cloud out of which they condensed. Consequently, all stars rotate. Stars spend the prevailing part of their lives as main-sequence (MS) objects fusing hydrogen in their cores. During the MS phase, the angular momentum of stars without mass-loss is conserved. This implies that stellar rotation period changes should occur on the timescale of the moment of inertia variations, which is of the order of $10^{7}-10^{9}$ years (Meynet \& Maeder 2000). How do we test this?

The magnetic chemically peculiar (mCP) stars, which have an abnormal surface chemical composition, are the most suitable test beds for studying the rotational evolution in upper MS

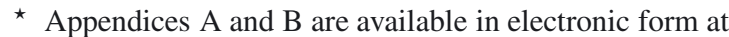
http://www. aanda.org stars. The overabundant elements in their atmospheres are concentrated into large, persistent spot regions. As the star rotates, periodic variations in the brightness, spectrum, and magnetic field are observed. Combining both present and archival observations of $\mathrm{mCP}$ stars collected over the past several decades, we can reconstruct their rotational evolution with unprecedented accuracy.

Careful period analyses of photometric observations of dozens of the best-monitored mCP stars listed in the on-line catalogue of photometric observations of magnetic chemically peculiar stars (Mikulášek et al. 2007) have been performed in Mikulášek et al. (2011). They confirm our theoretical expectations that the rotation periods and light curves of most upper MS stars are constant on timescales of decades. A few mCP stars display secular cyclic changes in the shape of their light curves (see e.g., Žižňovský et al. 2000), which can be attributed to the 
precession of magnetically distorted stars (Pyper \& Adelman 2004). However, there is also a small subgroup of hot mCP stars that have stable light curve shapes and spectroscopic variability, but exhibit variable rotation periods (Pyper et al. 1998; Mikulášek et al. 2008; Townsend et al. 2010). Constancy of their light curves on the scale of decades excludes precession as the cause of the observed period changes (for details see Mikulášek et al. 2008).

This letter concentrates on the period analysis of the bestmonitored mCP stars - CU Virginis and V901 Orionis.

\section{The stars}

\subsection{CU Virginis}

CU Vir = HD $124224=$ HR 5313 is one of the most enigmatic stars in the upper MS. This very fast rotating silicon $\mathrm{mCP}$ star has a moderate, nearly dipolar magnetic field (Borra \& Landstreet 1980; Pyper et al. 1998) with the pole strength of $B_{\mathrm{p}}=3.0 \mathrm{kG}$ tilted towards the rotational axis by $\beta=74^{\circ}$, the axis inclination being $i \simeq 43^{\circ}$ (Trigilio et al. 2000). CU Vir is the only known MS star that shows variable radio emission, resembling the radio lighthouse of pulsars (Trigilio et al. 2008, 2011; Ravi et al. 2010), it also displays variations in light and spectral lines of $\mathrm{He}$ I, Si II, H I, and other ions. The nature of its variability is minutely studied in Krtička et al. (2011). CU Vir belongs to the most frequently studied mCP stars.

Occasional rapid increases in its rotation period have been reported and discussed. Pyper et al. $(1997,1998)$ discovered an abrupt increase of the period from 0.5206778 to 0.52070854 that occurred approximately in 1984 and Pyper \& Adelman (2004) discussed two possible scenarios of the explanation of the observed O-C diagram, namely a continually changing period or two constant periods. After the year 1998 Trigilio et al. (2008, 2011) observed another increase in the period of radio pulses of $\Delta P=1.12 \mathrm{~s}$ with respect to the period determined by Pyper et al. (1998). However, the 2010 measurements indicate some period decrease. The rate of the deceleration can be evaluated using the spin-down time, $\tau$, defined as $\tau=P / \bar{P}$, where $P(t)$ is the instantaneous rotation period at the time $t$ and $\bar{P}$ is the mean rate of the rotational deceleration. The paradox of CU Vir is according to Mikulášek et al. (2011) that its spin down time, $\tau \sim 6 \times 10^{5}$ years, is more than two orders of magnitude shorter than the estimated age of the star $-9 \times 10^{7} \mathrm{yr}$ (Kochukhov \& Bagnulo 2006).

\subsection{V901 Orionis}

V901 Ori $=$ HD 37776 is a young hot star (B2 IV) residing in the emission nebula IC 432, with a global, extraordinarily strong $\left(B_{\mathrm{S}} \approx 20 \mathrm{kG}\right)$, and unusually complex magnetic field (Thompson \& Landstreet 1985; Kochukhov et al. 2011). The observed moderate light variations are caused by the spots of overabundant silicon and helium (Krtička et al. 2007).

Three decades of precise photometric and spectroscopic monitoring have revealed a continuous rotational deceleration (Mikulášek et al. 2008), increasing the period of about 1.5387 by a remarkable $18 \mathrm{~s}$ ! Ruling out (a) the light-time effect in a binary star; (b) the precession of the star's rotational axis; and (c) evolutionary effects as possible causes of the period change, we interpreted the deceleration in terms of the rotational braking of the outer stellar layers as caused by the angular momentum loss in the stellar magnetosphere.

However, this cannot explain the discrepancy between the spin-down time, $\tau=2.5 \times 10^{5} \mathrm{yr}$, and the star's age of one million
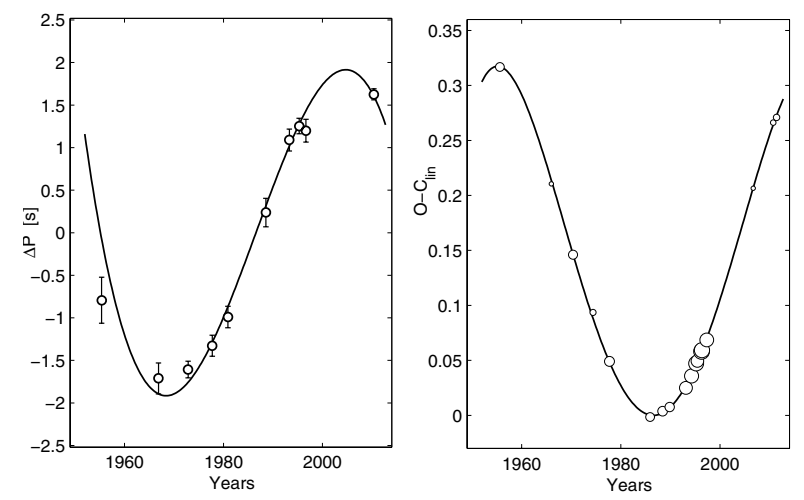

Fig. 1. Long-term variations of the rotation period and O-C values for the magnetic chemically peculiar star CU Vir. Left: the model curve of long-term rotational period changes $\Delta P$ of CU Vir in seconds with respect to the mean period $P_{0}=0.52069415$. The formal accuracy of the polynomial fit is comparable with the thickness of the fitted line. Right: the difference of the observed and calculated times of zero phase according to a linear ephemeris in days. Each point represents the average of 498 consecutive individual measurements; the weights of these means are indicated by their areas.

years or older (Mikulášek et al. 2008, 2010, 2011; Kochukhov $\&$ Bagnulo 2006). The interpretation of the rotation period evolution by simple angular motion loss was also questioned by the negative value of the second derivative of the period: $\ddot{P}=$ $-29(13) \times 10^{-13} \mathrm{~d}^{-1}$, which indicated that braking could change into acceleration (Mikulášek et al. 2008). Nevertheless, the significance of this conclusion was fairly low.

\section{Observations}

For the period analyses of CU Vir we obtained some new photometric or spectroscopic data, specifically: 1) JK derived 210 individual spectrophotometric magnitudes in 21 bands (201$299 \mathrm{~nm}$ ) by processing 10 IUE spectra, in 1979 January-March (for details see Krtička et al. 2011); 2) GWH obtained 374 precise $B V$ measurements at Fairborn Observatory Arizona, USA, in 2010 February-2011 June; 3) JL obtained $38 \mathrm{~V}$ measurements using his own microtelescope at the SAAO, South Africa and Brno, CR, in 2010 April-June; 4) JJ obtained $251 v b$ observations at Suhora Observatory, Poland, in 2011 March; and JJ+JL obtained $536 U B V$ measurements using the $0.5 \mathrm{~m}$ reflector of the SAAO, South Africa, in 2011 May.

We collected a total of 8965 individual measurements of CU Vir obtained between 1949 and 2011 (62 years or 43662 revolutions of the star) including 8270 photometric and spectrophotometric measurements in photometric bands from 200 to 753 $\mathrm{nm}$ as well as spectroscopic, spectropolarimetric and radiometric observations. The diverse observational data originated from 39 sources (see the list in Appendix B).

The V901 Ori data listed in Mikulášek et al. (2008) were enhanced with 98 precise $B V$ measurements obtained by GWH at Fairborn Observatory Arizona, USA from September 2009 to February 2011. We used a total of 2611 individual measurements obtained from 1976 to 2011 from 14 sources. In addition to 2409 photometric measurements, the data include 202 measurements of equivalent widths of eleven selected He I lines.

\section{The models and their results}

The method of data processing, outlined in the Appendix A, assumes that phase curves of all monitored quantities are constant, 


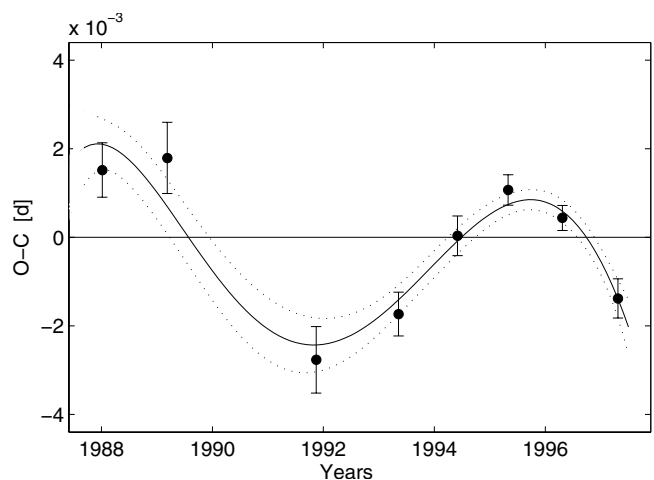

Fig. 2. Medium-term variations in the rotational period of CU Vir. The modulation of the long-term rotational changes in the observed time of the light minimum minus the calculated time of light minimum is expressed in days. Owing to uneven sampling within the observing seasons, we were not able to detect rotational modulation on shorter timescales.

while the "instant period" of changes $P(t)$ is variable in time $t$. It is advantageous to introduce a "phase function" $\vartheta(t, \boldsymbol{b})$, which is a continuous monotonic function of time $t$. The fractional part of it corresponds to the common phase $\varphi$, the integer part is the socalled epoch $(E)$ and $\boldsymbol{b}$ is a set of $g_{\mathrm{b}}$ free parameters describing a model of the period development. Quantities $P(t)$ and $\vartheta(t, \boldsymbol{b})$ are bound by the simple equality $P(t)=1 / \dot{\vartheta}$, where the dot refers to the first time derivative of the quantity. Because we did not know the true nature of observed period changes of $P(t)$ we chose standard low-orders polynomials for their description.

\subsection{CU Virginis}

The period changes $P(t)$ (see Fig. 1 ) can be adequately approximated by a cubic parabola with the origin at the time $t=T_{0}$,

$$
P(t)=P_{0}+A\left(3 \Theta-4 \Theta^{3}\right) ; \quad \Theta=\left(t-T_{0}\right) / \Pi,
$$

where $A$ and $\Pi$ are parameters expressing the amplitude and timescale of the observed period changes, and $\Theta$ is a time-like function. The instant period $P(t)$ reaches its local extrema $P_{0} \mp A$ at $\Theta_{1,2}=\mp \frac{1}{2}$, where $P_{0}$ is the period at the time of $t(\Theta=0)=T_{0}$. The value $\Pi$ then equals the duration of the rotation deceleration epoch that took place in the time interval $t_{1,2}=T_{0} \mp \frac{1}{2} \Pi$.

Applying the equality $\dot{\vartheta}=1 / P(t)$, we obtain the following approximation for the phase function in the form of the fourthorder polynomial of time

$\vartheta(t, \mathbf{b}) \cong \vartheta_{0}-\frac{B}{P_{0}}\left(\frac{3}{2} \Theta^{2}-\Theta^{4}\right) ; \vartheta_{0}=\frac{t-M_{0}}{P_{0}}, B=\frac{A \Pi}{P_{0}}$,

where $\vartheta_{0}$ is the phase function for a linear ephemeris with the origin at $M_{0} \equiv 2446730.4447$ and the basic period $P_{0}$.

Using the above formulated phenomenological model, we derive $P_{0}=0.52069415(8), \Pi=13260(70) \mathrm{d}=36.29(19)$ $\mathrm{yr}, T_{0}=2446636(24)$ or $1986.56(7), A=1.915(10) \mathrm{s}$, and $B=0.5643(29) \mathrm{d}$. The period $P(t)$ reached its local minimum in the year 1968.4, $P_{\min }=0.52067198(7)$, and its local maximum in 2004.7: $P_{\max }=0$ d52071631(18). The rotational deceleration rate reached its maximum $\dot{P}=0.158 \mathrm{~s} \mathrm{yr}^{-1}$ in the year 1986.6. The zero phase times of CU Vir can be evaluated using the relation $J D(k) \cong M_{0}+P_{0} k+B\left(\frac{3}{2} \Theta_{k}^{2}-\Theta_{k}^{4}\right)$, where $k$ is an integer and $\Theta_{k}=\left(M_{0}+P_{0} k-T_{0}\right) / \Pi$.
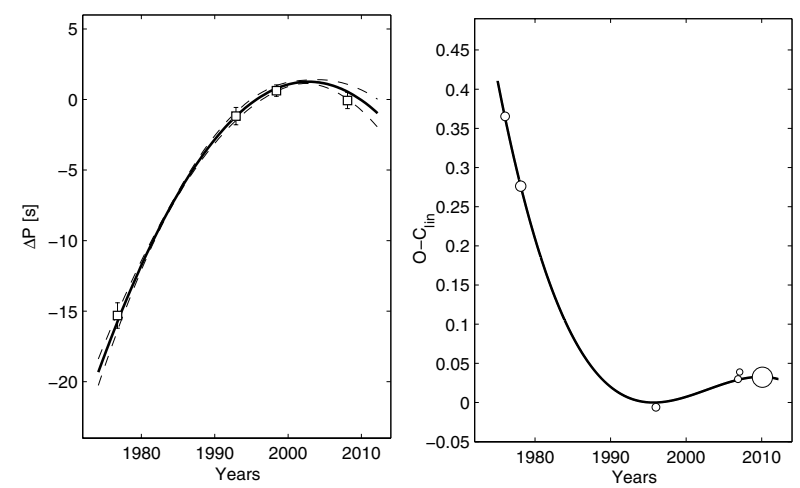

Fig. 3. Long-term variations in the rotational period and O-C diagram of V901 Ori. Left: variation in the rotation period $\Delta P$ in seconds with respect to the mean period $P_{0}=1.538756(3)$, derived from 2611 individual measurements. The $1-\sigma$ deflections from the quadratic fit are denoted by dashed lines. Points with error bars correspond to virtual period deflections for appropriately selected groups of consecutive individual data items. Right: the difference of the observed zero phase time and the time calculated according to the linear ephemeris in fractions of a day. Each point represents an average of 435 consecutive measurements; the weights of these means are indicated by their areas.

The basic model fits the observed long-term period changes very well and enables us to predict the zero phase times with an accuracy of $0.001 \mathrm{~d}$. However, the analysis of the residuals from the accepted polynomial model reveals an additional variation. Between 1988 and 1998, for which there is excellent photometric coverage (Adelman et al. 1992; Pyper et al. 1998), we find a small modulation of the period on a timescale of several years (Fig. 2).

\subsection{V901 Orionis}

For the phase function of V901 Ori we adopted polynomials in the form of the third-order Taylor-expansion, also used in Mikulášek et al. (2008):

$\vartheta(t, \boldsymbol{b})=\vartheta_{0}-\frac{1}{2} \dot{P}_{0} \vartheta_{0}^{2}-\frac{1}{6} P_{0} \ddot{P} \vartheta_{0}^{3} ; \quad \vartheta_{0}=\left(t-M_{0}\right) / P_{0} ;$

$P(t)=1 / \dot{\vartheta} \cong P_{0}\left(1+\dot{P}_{0} \vartheta_{0}+\frac{1}{2} P_{0} \ddot{P} \vartheta_{0}^{2}\right)$,

where $\vartheta_{0}$ is the phase function for the linear ephemeris with the origin at $M_{0} . P_{0}$ and $\dot{P}_{0}$ are the instantaneous period and its first derivative at $M_{0}$. We assumed that the second derivative of the period, $\ddot{P}$, is constant throughout the interval of observations.

We put the origin, $M_{0}=2449967.969(5)$, at a brightness maximum (in 1999). Our fitted parameter values include $P_{0}=1.538756(3)$, the instantaneous period at time $M_{0}$, and $\dot{P}_{0}=11.3(0.7) \times 10^{-9}=0.356(23) \mathrm{s} \mathrm{yr}^{-1}$ and $\ddot{P}=-4.4(6) \times$ $10^{-12} \mathrm{~d}^{-1}=-1.38(19) \times 10^{-5} \mathrm{~s} \mathrm{yr}^{-2}$, the first and second time derivatives. The negative value of $\ddot{P}$ is now established with 7- $\sigma$ certainty. Accordingly, the star reached its longest period, $P_{\max }=1.5387709(15)$, in $2002.8 \pm 1.1$, and is now accelerating again (see Fig. 3). The zero-phase timing can be calculated by the relation $J D(k) \cong M_{0}+P_{0}\left(k+\frac{1}{2} \dot{P}_{0} k^{2}+\frac{1}{6} P_{0} \ddot{P} k^{3}\right)$, where $k$ is an integer. There is no indication of any short-term period modulation analogous to that seen in CU Vir.

\section{Discussion}

We were able to reveal unexpected alternating lengthenings and shortenings in the periods of CU Vir and V901 Ori owing to simultaneous processing of all available observational material obtained during many decades, including our own observations 
made in 2007-2011. The period evolutions of both stars were modelled by polynomials of low degrees. In the case of CU Vir we also tested a fit with a harmonic function (Mikulášek et al. 2011) and a Gaussian function corresponding to the transient nature of the phenomenon. The only differences among them are noticeable at the very beginning of our observations, which slightly favour the latter two alternatives.

The possible harmonic-like changes of the observed periods of both stars together with the strict constancy of the phase curves can in principle be explained by the light time effect caused by an invisible companion orbiting the star in a nearly circular orbit. However, this orbital motion would be revealed by considerable radial velocity variations $\Delta R V$. In the case of CU Vir the observed change in the period $\Delta P$ of $3.8 \mathrm{~s}$ corresponds to a variation $\triangle R V=c \Delta P / P=25 \mathrm{~km} \mathrm{~s}^{-1}$, while the observed change in V901 Ori period of $\Delta P \simeq 20 \mathrm{~s}$ predicts a $\Delta R V$ variation of even $45 \mathrm{~km} \mathrm{~s}^{-1}$ ! However, no such longterm $R V$ variations synchronised with period changes have been found (Pyper et al. 1997; Mikulášek et al. 2008). Additionally, the light time effect cannot explain the complex medium-term period variations observed in CU Vir (see Sect. 4.1, Fig. 2).

We conclude that the observed period variations are caused by uneven rotation of surface layers of the stars (Stępień 1998; Mikulášek et al. 2008, 2011). However, the physical explanation of the observed rotation period variations is not straightforward. Rotational braking by angular momentum (AM) loss via a magnetised stellar wind (Mikulášek et al. 2008) cannot be the sole cause of the period variations because we detected intervals when the rotational period decreases. Consequently, the AM loss may affect only the outer stellar envelope that is hardened by the global magnetic field, leaving the faster rotating core unaffected. Intervals of rotational deceleration may then alternate with intervals of angular momentum exchange between the slowly rotating envelope and the faster rotating core. The latter manifests itself by the rotational acceleration of the outer envelope.

However, this explanation is theoretically challenging. Given the intermediate age of $\mathrm{CU}$ Vir, the detected rotation variations are likely not connected with the transient behaviour that is connected with settling into the MS stage. Therefore, we can expect a stable inner configuration in both stars. As follows from Flowers \& Ruderman (1977) and Braithwaite \& Nordlund (2006), the magnetic fields confined to the outer stellar layers only are unstable. On the other hand, a magnetic field that penetrates deep into a star causes nearly uniform rotation (Moss et al. 1990; Charbonneau \& MacGregor 1992; Maeder \& Meynet 2003).

The possible role of stellar winds in the mechanism of the rotational instability is supported by the fact that stronger period variations are detected in V901 Ori, which is more luminous than CU Vir and, consequently, has a stronger stellar wind (Krtička \& Kubát 2001). Moreover, this could help to explain the fact that the younger star V901 Ori rotates more slowly than CU Vir.

All these considerations should be the subject of additional physical modelling. Nevertheless, the aim of this letter is more unpretentious - we only want to draw the attention to an interesting property of the rotation of some upper MS stars.

\section{Conclusions}

Our study investigated the nature of the rotational period variations in two well-observed mCP stars CU Vir and V901 Ori. Contrary to the results of previous studies, we show that the period changes are not monotonic - intervals of rotational deceleration alternate with intervals of rotational acceleration, all on the timescale of several decades. These results explain the spin-down time paradox of these stars, according to which the spin-down time was significantly shorter than the age of these stars. On the other hand, this unexpected behaviour of two fairly dissimilar $\mathrm{mCP}$ stars poses a strong challenge for any theoretical models.

Acknowledgements. We thank R. von Unge, D. M. Pyper, M. Lenc, S. J. Adelman, T. Ryabchikova, and R. Arlt for valuable discussions and unpublished observational data. This work was funded by the following grants: GAAV IAA301630901, GAČR 205/08/H005, MEB 061014/WTZ CZ 10-2010, MEB 0810095, VEGA 2/0074/09, and MUNI/A/0968/2009. We also thank the referee D. Moss for valuable comments and inspiring suggestions.

\section{References}

Abt, H. A., \& Snowden, M. S. 1973, A\&AS, 25, 137

Abuladze, O. P. 1968, Bjul. Abustam. Astrofiz. Obs., 36, 43

Adelman, S. J., Dukes, R. J. Jr., \& Pyper, D. M. 1992, AJ, 104, 314

Blanco, C., \& Catalano, F. 1971, AJ, 76, 630

Borra, E. F., \& Landstreet, J. D. 1980, ApJS, 42, 421

Braithwaite, J., \& Nordlund, A. 2006, A\&A, 450, 1077

Charbonneau, P., \& MacGregor, K. B. 1992, ApJ, 387, 639

Deutsch, A. 1952, ApJ, 116, 356

ESA, 1998, The Hipparcos and Tycho Catalogs, Celestia 2000, SP-1220

Flowers, E., \& Ruderman, M. A. 1977, ApJ, 215, 302

Hardie, P., 1958, ApJ, 127, 620

Hardie, R. H., Lieberman, L., \& Reichmann, E. 1967, PASP, 79, 74

Hardorp J., \& Megessier C. 1977, A\&A, 61, 411

Hiesberger F., Piskunov T. A., Bonsack W. K., et al. 1995, A\&A, 296, 473

Kochukhov, O., \& Bagnulo, S. 2006, ApJ, 726, 24

Kochukhov, O., Lundin, A., Romanyuk, I., \& Kudryavtsev, D. O. 2011, A\&A, 450,763

Krivosheina, A. A., Ryabchikova, T. A., \& Khokhlova, V. L. 1980, Nauchnii Inform. Astron. Council, Ser. Astrofizika, No. 43, 70 (in Russian)

Kuschnig, R., Ryabchikova, T. A., Piskunov, N. E., et al. 1999, A\&A, 348, 924

Krtička, J., \& Kubát, J. 2001, A\&A, 369, 222

Krtička, J., Mikulášek, Z., Zverko J., \& Žižňovský J. 2007, A\&A, 470, 1089

Krtička, J., Mikulášek, Z., Lüftinger, T., et al. 2011, A\&A, submitted

Landstreet, J. D., \& Borra, E. F. 1978, ApJ, 224, 5

Meynet, G., \& Maeder, A. 2000, A\&A, 361, 101

Maeder, A., \& Meynet, G. 2003, A\&A, 411, 543

Mikulášek, Z., Janík, J., Zverko, J. et al. 2007, Astron. Nachr., 328, 10

Mikulášek, Z., Krtička, J., Janík J. et al. 2011 [arXiv: 1106.4258]

Mikulášek, Z., Krtička, J., Henry, G. W. et al. 2008, A\&A, 485, 585

Mikulášek, Z., Krtička, J., Henry, G. W. et al. 2010, A\&A, 511, L7

Molnar, M. R., \& Wu C.-C. 1978, A\&A, 63, 335

Moss, D. L., Mestel, L., \& Tayler, R. J. 1990, MNRAS, 245, 550

Musiełok B., Ryabchikova, T. L., Davydova, \& Madej J. 1990, Mitt. KarlSchwarzschild-Obs. Tautenburg No. 125, 110

Pedersen, H. 1978, A\&AS, 33, 203

Peterson, B. A. 1996, ApJ, 145, 735

Pojmański, G. 2001, ASP Conf. Ser. 246, IAU Colloquium 183, ed. B. Paczynski, W.-P. Chen, \& C. Lemme, 53

Pyper, D. M., \& Adelman, S. J. 1985, A\&AS, 59, 369

Pyper, D. M. \& Adelman, S. J. 2004, The A-Star Puzzle, ed. J. Zverko, J. Žižňovský, S. J. Adelman, \& W. W. Weiss (Cambridge University Press, Cambridge), IAU Symposium, 224, 307

Pyper, D. M., Ryabchikova, T., \& Malanushenko, V. 1997, BAAS, 29, 811

Pyper, D. M., Ryabchikova, T., Malanushenko, V., et al. 1998, A\&A, 339, 822

Ravi, V., Hobbs, G., Wickramasinghe, D., Champion, D. J., \& Keith M. 2010, MNRAS, 408, 99

Stępień, K. 1998, A\&A, 337, 754

Thompson, I. B., \& Landstreet, J. D. 1985, ApJ, 289, 9

Townsend, R. H. D., Oksala, M. E., Cohen, D. H., Owocki, S. P., \& ud-Doula, A. 2010, ApJ, 714, 318

Trigilio, C., Leto, P., Leone, F., Umana, G., \& Buemi, C. 2000, A\&A, 362, 281

Trigilio, C., Leto, P., Umana, G., Buemi, C. S., \& Leone, F. 2008, MNRAS, 384, 1437

Trigilio, C., Leto, P., Umana, G., Buemi, C. S., \& Leone, F. 2011, ApJ, 739, L10

Weiss, W. W., Albrecht, R., \& Wieder R. 1976, A\&A, 47, 423

Winzer, J. E. 1974, Ph.D. Thesis, Univ. Toronto

Žižňovský, J., Schwartz, P., \& Zverko, J. 2000, IBVS, 4835 


\section{Appendix A: The outline of the method}

The techniques used to analyse the data are based on the rigourous application of non-linear, weighted, least-squares methods used simultaneously for all relevant data that contain phase information. Our technique does not use an O-C diagram as an intermediate stage of data processing; O-C diagrams are used only as a visual check on the adequacy of the models. Let us assume that all observed phase curves of a star are adequately described by the unique general model function $F(\vartheta, \boldsymbol{a})$, described here by $g_{a}$ parameters contained in a parameter vector $\boldsymbol{a}, \boldsymbol{a}=\left(a_{1}, \ldots, a_{j}, \ldots, a_{g_{a}}\right)$. In our computation we assume that the form of all the phase variations is constant and that the time variability of the observed quantities are given by a phase function $\vartheta(t, \boldsymbol{b})$, which is a monotonic function of time $t$. The fractional part of it corresponds to the common phase, the integer part is the so-called epoch $(E)$. We can express the phase function by means of a simple model quantified by $g_{b}$ parameters $\boldsymbol{b}$, $\boldsymbol{b}=\left(b_{1}, \ldots, b_{k}, \ldots, b_{g_{b}}\right)$. The instantaneous period is given simply by the equality $P(t)=1 / \dot{\vartheta}$.

For a realistic modelling of phase variations for all data types for a star, we need $g_{a}$ free parameters for the description of the model function $F(\vartheta, \boldsymbol{a})$ and $g_{b}$ free parameters for the description of the phase function $\vartheta(t, \boldsymbol{b})$. The computation of the free parameters was iterative under the basic condition that the weighted sum $S(\boldsymbol{a}, \boldsymbol{b})$ of the quadrates of the difference $\Delta y_{i}$ of the observed value $y_{i}$ and its model prediction is minimal ( $w_{i}$ being the individual weight of the $i$-th measurement).

$\Delta y_{i}=y_{i}-F\left(\vartheta_{i}\right) ; \quad S=\sum_{i=1}^{n} \Delta y_{i}^{2} w_{i} ; \quad \delta S=\mathbf{0} ; \quad \Rightarrow$
$\sum_{i=1}^{n} \Delta y_{i} \frac{\partial F\left(\vartheta_{i}, \boldsymbol{a}\right)}{\partial a_{j}} w_{i}=0 ; \quad \sum_{i=1}^{n} \Delta y_{i} \frac{\partial F}{\partial \vartheta_{i}} \frac{\partial \vartheta\left(t_{i}, \boldsymbol{b}\right)}{\partial b_{k}} w_{i}=0$.

We obtain here $g=g_{a}+g_{b}$ equations of $g$ unknown parameters. The weights of individual measurements $w_{i}$ are inversely proportional to their expected uncertainty. The system is non-linear; we have to determine the parameters iteratively. With a good initial estimate of the parameter vectors $a$ and $b$, the iterations converge very quickly. Usually we need only several tens of iterations to complete the iteration procedure.

\section{A.1. Virtual O-C diagrams. Evolution of periods}

The short-term modulation of the phase function was analysed by means of the residuals of the observed data $\Delta y_{i}$, creating individual values of the phase shifts expressed in days $(\mathrm{O}-\mathrm{C})_{j}$ with adapted individual weight $W_{j}$ for each observed datum and averages of the phase shifts defined for arbitrarily selected groups of measurements ${\overline{(\mathrm{O}-\mathrm{C})_{i}}}_{i}$ or deflection of the mean period from the instant model period $\Delta P_{k}\left(t_{k}\right)$ :

$$
\begin{aligned}
& (\mathrm{O}-\mathrm{C})_{j}=-P\left(t_{j}\right) \Delta y_{j}\left(\frac{\partial F}{\partial \vartheta}\right)^{-1} ; \quad W_{j}=\left(\frac{\partial F}{\partial \vartheta}\right)^{2} w_{j} ; \\
& \overline{(\mathrm{O}-\mathrm{C})_{k}}=\frac{\sum_{j=1}^{n_{k}}(\mathrm{O}-\mathrm{C})_{j} W_{j}}{\sum_{j=1}^{n_{k}} W_{j}} ; \quad \Delta P_{k}=\frac{\sum_{j=1}^{n_{k}}(\mathrm{O}-\mathrm{C})_{j} \vartheta_{j} W_{j}}{\sum_{j=1}^{n_{k}} \vartheta_{j}^{2} W_{j}} .
\end{aligned}
$$

Computations of ${\overline{(\mathrm{O}-\mathrm{C})_{k}}}_{k}$ and $\Delta P_{k}$ followed after the model parameters were found; consequently they had no influence on the model solution. They were used only to visualise of the solution.
Table B.1. Data with phase information about CU Vir used in the period analysis.

\begin{tabular}{llrl}
\hline \hline Season/s & Data type & $N$ & Reference \\
\hline $1949-52$ & EW He I & 34 & Deutsch (1952) \\
1955 & $U B V$ & 162 & Hardie (1958) \\
$1964-6$ & $U B V$ & 702 & Abuladze (1968) \\
1964 & EW Si II & 40 & Peterson (1996) \\
1964 & EW He I & 9 & Peterson (1996) \\
$1965-6$ & RV H $\gamma$ & 16 & Abt \& Snowden (1973) \\
1966 & $B$ & 33 & Hardie et al. (1967) \\
1967,76 & EW He I & 37 & Hardorp \& Megessier (1977) \\
1967,76 & EW Si II & 19 & Hardorp \& Megessier (1977) \\
$1968-9$ & EW Si II & 124 & Krivosheina et al. (1980) \\
$1968-9$ & EW H $\gamma$ & 21 & Krivosheina et al. (1980) \\
1968 & UBV & 287 & Blanco \& Catalano (1971) \\
$1972-81$ & spf mag & 179 & Pyper \& Adelman (1985) \\
1972 & $U B V$ & 29 & Winzer (1974) \\
1974 & $\beta$ & 95 & Weiss et al. (1976) \\
1974 & $u v b y W$ & 460 & Weiss et al. (1976) \\
1975 & spf OAO II & 54 & Molnar \& Wu (1978) \\
$1976-8$ & $B_{\text {eff }}$ & 14 & Borra \& Landstreet (1980) \\
1977 & EW He I & 69 & Pedersen (1978) \\
1979 & spf IUE & 210 & Krtička et al. (2011) \\
$1980-3$ & $u v b y$ & 89 & Pyper \& Adelman (1985) \\
$1980-3$ & $\beta$ & 23 & Pyper \& Adelman (1985) \\
1983 & EW He I & 9 & Hiesberger et al. (1995) \\
$1987-9$ & $U B V$ & 1012 & Adelman et al. (1992) \\
1988 & $\beta$ & 25 & Musiełok et al. (1990) \\
$1990-3$ & $B_{\mathrm{T}} V_{\mathrm{T}} H_{\mathrm{p}}$ & 288 & ESA (1998) \\
$1991-7$ & $u v b y$ & 3635 & Pyper et al. (1998) \\
$1994-5$ & EW He I & 19 & Kuschnig et al. (1999) \\
1995 & RV H $\delta$ & 19 & Kuschnig et al. (1999) \\
1995 & EW Si II & 19 & Kuschnig et al. (1999) \\
1995 & EW H $\delta$ & 18 & Kuschnig et al. (1999) \\
1995 & $B_{\text {eff }}$ & 21 & Pyper et al. (1998) \\
$1998-09$ & radio & 5 & Ravi et al. (2010) \\
$2002-9$ & $V$ & 353 & Pojmański et al. (2001) \\
$2010-11$ & $B V$ & 374 & Henry - this paper \\
$2010-11$ & EW Si II & 59 & Janík - this paper \\
2010 & $V$ & 38 & Liška - this paper \\
2011 & $v b$ & 251 & Janík - this paper \\
2011 & $U B V$ & 402 & Janík \& Liška - this paper \\
\hline & & &
\end{tabular}

Similarly, we can compute virtual "observed" values of the instant period from a group of observations to generate the model curves in our figures.

Relation A.1 can also be used to determine reliably zerophase times for selected groups of observations $\mathrm{O}_{k}$. These quantities depend only marginally on the chosen model of the phase function $\vartheta(t, \boldsymbol{b})$. Therefore we can use these $\mathrm{O}_{k}$ in the process of the phase function modelling.

\section{Appendix B: Brief specification of CU Vir data}

The data used for the analysis of CU Vir are given in Table B.1. Here we used the following abbreviations: EW - the equivalent width, $\mathrm{RV}$ - radial velocity, $\beta-\mathrm{H} \beta$ photometry, $U B V$ - Johnson $U B V$ photometry, $u v b y$ - Stroemgren $u v b y$ photometry, $B_{\mathrm{T}} V_{\mathrm{T}} H_{\mathrm{p}}$ - Hipparcos photometry, $B_{\text {eff }}$ - the mean longitudinal magnetic induction, $\mathrm{spf}$ - magnitudes derived from spectrograms obtained by UV satellites OAO 2 and IUE, and radio - timings of radiopulses. 